\title{
Lowness, randomness, and computable analysis
}

\author{
André Nies \\ Department of Computer Science, University of Auckland
}

\begin{abstract}
Analytic concepts contribute to our understanding of randomness of reals via algorithmic tests. They also influence the interplay between randomness and lowness notions. We provide a survey.
\end{abstract}

\section{Introduction}

Our basic objects of study are infinite bit sequences, identified with sets of natural numbers, and often simply called sets. A lowness notion provides a sense in which a set $A$ is close to computable. For example, $A$ is computably dominated if each function computed by $\mathrm{A}$ is dominated by a computable function; $A$ is low if the halting problem relative to $A$ has the least possible Turing complexity, namely $A^{\prime} \equiv_{T} \varnothing^{\prime}$. These two notions are incompatible outside the computable sets, because every non-computable $\Delta_{2}^{0}$ set has hyperimmune degree.

Lowness notions have been studied for at least 50 years [51,33,27]. More recently, and perhaps suprisingly, ideas motivated by the intuitive notion of randomness have been applied to the investigation of lowness. On the one hand, these ideas have led to new lowness notions. For instance, $K$-triviality of a set of natural numbers (i.e., being far from random in a specific sense) coincides with lowness for Martin-Löf randomness, and many other notions. On the other hand, they have been applied towards a deeper understanding of previously known lowness notions. Randomness led to the study of an important subclass of the computably dominated sets, the computably traceable sets [52]. Superlowness of an oracle $A$, first studied by Mohrherr [36], says that $A^{\prime} \equiv_{t t} \varnothing^{\prime}$; despite the fact that the low basis theorem [27] actually yields superlow sets, the importance of superlowness was not fully appreciated until the investigations of lowness via randomness. For instance, every $K$-trivial set is superlow [39].

Computable analysis allows us to characterise several randomness notions that were originally defined in terms of algorithmic tests. Schnorr [49] introduced two randomness notions for a bit sequence $Z$ via the failure of effective betting strategies. Nowadays they are called computable randomness and Schnorr randomness. Computable randomness says that no 
effective betting strategy (martingale) succeeds on $Z$, Schnorr randomness that no such strategy succeeds quickly (see $[11,40]$ for background). Pathak [44], followed by Pathak, Rojas and Simpson [45] characterised Schnorr randomness: $Z$ is Schnorr random iff an effective version of the Lebesgue differentiation theorem holds at the real $z \in[0,1]$ with binary expansion $Z$. Brattka, Miller and Nies [6] showed that $Z$ is computably random if and only if every nondecreasing computable function is differentiable at $z$. See Section 4 for detail.

From 2011 onwards, concepts from analysis have also influenced the interplay of lowness and randomness. The Lebesgue density theorem for effectively closed sets $\mathcal{C}$ provides two randomness notions for a bit sequence $Z$ which are slightly stronger than Martin-Löf's. In the stronger form, the density of any such set $\mathcal{C}$ that contains $Z$ has to be 1 at $Z$; in the weak form, the density is merely required to be positive. One has to require separately that $Z$ is ML-random (even the stronger density condition doesn't imply this, for instance because a 1-generic also satisfies this condition). These two notions have been used to obtain a Turing incomplete Martin-Löf random above all the K-trivials, thereby solving the so-called ML-covering problem. We give more detail in Section 5; also see the survey [3].

In current research, concepts inspired by analysis are used to stratify lowness notions. Cost functions describe a dense hierarchy of subideals of the K-trivial Turing degrees (Section 6). The Gamma and Delta parameters are real numbers assigned to a Turing degree. They provide a coarse measure of its complexity in terms of the asymptotic density of bit sequences (Section 7).

The present paper surveys the study of lowness notions via randomness. In Sections 2 and 3 we elaborate on the background in lowness, and how it was influenced by randomness. Section 4 traces the interaction of computable analysis and randomness from Lebesgue to the present. Section 5 shows how some of the advances via computable analysis aided the understanding of lowness through randomness. Sections 6 and 7 dwell on the most recent developments. A final section contains open questions.

\section{Early days of lowness}

Spector [51] was the first to construct a Turing degree that is minimal among the nonzero degrees. Sacks [47] showed that such a degree can be $\Delta_{2}^{0}$. Following these results, as well as the Friedberg-Muchnik theorem and Sacks' result [48] that the c.e. Turing degrees are dense, the 
interest of early computability theorists focussed on relative complexity of sets: comparing their complexity via an appropriate reducibility. Absolute complexity, which means finding natural lowness classes and studying membership in them, received somewhat less attention, and was mostly restricted to classes defined via the Turing jump. Martin and Miller [33] built a perfect closed set of computably dominated oracles. Jockusch and Soare [27] proved that every non-empty effectively closed set contains a low oracle. These constructions used recursion-theoretic versions of forcing. Jockusch published papers such as $[26,25]$ that explored notions such as degrees of diagonally noncomputable functions, and degrees of bi-immune sets. Downey's work in the 1980s was important for the development of our understanding of lowness. For instance, Downey and Jockusch [15] studied complexity of sets, both relative and absolute, using ever more sophisticated methods.

\section{Randomness interacts with lowness}

We begin with the following randomness notions:

weakly 2-random $\rightarrow$ ML-random $\rightarrow$ computably rd. $\rightarrow$ Schnorr rd.

$Z$ is weakly 2-random iff $Z$ is in no null $\Pi_{2}^{0}$ class. Section 5 will develop notions implied by weak 2-randomness, and somewhat stronger than MLrandomness.

Lowness can be used to understand randomness via the randomness enhancement principle [41], which says that sets already enjoying a randomness property get more random as they become lower in the sense of computational complexity. Every non-high Schnorr random is MLrandom. A ML-random is weakly 2-random iff it forms a Turing minimal pair with $\varnothing^{\prime}$. See $[11,40]$.

Here we are mostly interested in the converse interaction: studying lowness via randomness. Let $K(x)$ denote the prefix free version of Kolmogorov complexity of a binary string $x$. The $K$-trivial sets were introduced by Chaitin [8] and studied by Solovay in an unpublished manuscript [50], rediscovered by Calude in the 1990s. Most of this manuscript is covered in Downey and Hirschfeldt's monumental work [11]. We say that $A$ is $K$-trivial if $\exists b \forall n K(A \mid n) \leqslant K(n)+b$. By the Levin-Schnorr characterisation, $Z$ is ML-random iff $\exists d \forall n K(Z \mid n) \geqslant n-d$. Since $K(n) \leqslant \log _{2} n+O(1)$, this definition says that $K$-trivials are far from random. Each computable set is $K$-trivial; Solovay built a $K$-trivial $\Delta_{2}^{0}$ set $A$ that is not computable. This was later improved to a c.e. set $A$ by 
Downey et al. [13], who used what became later known as a cost function construction.

An oracle $A$ is called low for a randomness notion $\mathcal{C}$ if every $\mathcal{C}$ random set is already $\mathcal{C}$-random relative to $A$. $K$-triviality appears to be the universal lowness class for randomness notions based on c.e. test notions. $A$ is $K$-trivial iff $A \in \operatorname{Low}(\mathrm{W} 2 \mathrm{R}, \mathrm{CR})$, namely every weakly 2 random set is computably random relative to $A$. This was shown by the author $[40,8.3 .14]$ extending the result that $\operatorname{Low}(\mathrm{W} 2 \mathrm{R}, \mathrm{MLR})$ coincides with $K$-triviality [14]. As a consequence, for any randomness notion $\mathcal{D}$ in between weak- 2 randomness and computable randomness, lowness for $\mathcal{D}$ implies $K$-triviality. For many notions, e.g. weak-2 randomness [14] and ML-randomness [39], the classes actually coincide.

Some of the $K$-trivial story, including the roles Downey, Hirschfeldt and the author have played in it, is vividly described in [42]. Background and more detailed coverage for the developments up to 2009 can be found in the aforementioned books $[11,40]$.

\section{Randomness and computable analysis}

We discuss the influence exerted by computable analysis on the study of randomness notions. Thereafter we will return to our main topic, lowness notions.

Analysis and ergodic theory have plenty of theorems saying that a property of being well-behaved holds at almost every point. Lebesgue proved that a function of bounded variation defined on the unit interval is differentiable at almost every point. He also proved the density theorem, and the stronger differentiation theorem, that now both bear his name. The density theorem says that a measurable set $\mathcal{C} \subseteq[0,1]$ has density one at almost every of its members $z$. To have density one at $z$ means intuitively that many points close to $z$ are also in $\mathcal{C}$, and this becomes more and more apparent as one "zooms in" on $z$ :

Definition 1. Let $\lambda$ denote Lebesgue measure on $\mathbb{R}$. We define the lower Lebesgue density of a set $\mathcal{C} \subseteq \mathbb{R}$ at a point $z$ to be the limit inferior

$$
\underline{\varrho}(\mathcal{C} \mid z):=\liminf _{|Q| \rightarrow 0} \frac{\lambda(Q \cap \mathcal{C})}{|Q|},
$$

where $Q$ ranges over open intervals containing $z$. The Lebesgue density of $\mathcal{C}$ at $z$ is the limit (which may not exist)

$$
\varrho(\mathcal{C} \mid z):=\lim _{|Q| \rightarrow 0} \frac{\lambda(Q \cap \mathcal{C})}{|Q|} .
$$


Note that $0 \leqslant \underline{\varrho}(\mathcal{C} \mid z) \leqslant 1$.

Theorem 2 (Lebesgue [31]). Let $\mathcal{C} \subseteq \mathbb{R}$ be a measurable set. We have $\underline{\varrho}(\mathcal{C} \mid z)=1$ for almost every $z \in \mathcal{C}$.

The Lebesgue differentiation theorem says that for almost every $z$, the value of an integrable function $g$ at $z \in[0,1]$ is approximated by the average of the values around $z$, as one zooms in on $z$. A point $z$ in the domain of $g$ is called a weak Lebesgue point of $g$ if

$$
\lim _{\lambda Q \rightarrow 0} \frac{1}{\lambda(Q)} \int_{Q} g d \lambda
$$

exists, where $Q$ ranges over open intervals containing $z$; we call $z$ a Lebesgue point of $g$ if this value equals $g(z)$.

Theorem 3 (Lebesgue [30]) Suppose $g$ is an integrable function on $[0,1]$. Then almost every $z \in[0,1]$ is a Lebesgue point of $g$.

Lebesgue [32] extended this result to higher dimensions, where $Q$ now ranges over open cubes containing $z$. Note that if $g$ is the characteristic function of a measurable set $\mathcal{C}$, then the expression (1) is precisely the density of $\mathcal{C}$ at $z$.

In ergodic theory, one of the best-known "almost everywhere" theorems is due to G. Birkhoff: intuitively, given an integrable function $g$ on a probability space with a measure preserving operator $T$, for almost every point $z$, the average of $g$-values at iterates, that is, $\frac{1}{n} \sum_{i<n} g\left(T^{i}(z)\right)$, converges. If $T$ is ergodic (i.e., all $T$-invariant measurable sets are null or co-null), the limit equals $\int g$; in general the limit is given by the conditional expectation of $g$ with respect to the $\sigma$-algebra of $T$-invariant sets.

The important insight is this: if the collection of given objects in an a.e. theorem is effective in some particular sense, then the theorem describes a randomness notion via algorithmic tests. Every collection of effective objects constitutes a test, and failing it means to be a point of exception for this collection. Demuth [10] (see below) made this connection in the setting of constructive mathematics. In the usual classical setting, V'yugin [53] showed that Martin-Löf randomness of a point $z$ in a computable probability space suffices for the existence of the limit in Birkhoff's theorem when $T$ is computable and $g$ is $L_{1}$-computable. Here $L_{1}$-computability means in essence that the function can be effectively approximated by step functions, where the distance is measured in the usual $L_{1}$-norm. About ten years later, Pathak [44] showed that ML-randomness of $z$ suffices for the existence of the limit in the Lebesgue differentiation 
theorem when the given function $f$ is $L_{1}$-computable. This works even when $f$ is defined on $[0,1]^{n}$ for some $n>1$. Pathak, Rojas and Simpson [45] showed that in fact the weaker condition of Schnorr randomness on $z$ suffices. They also showed a converse: if $z$ is not Schnorr random, then for some $L_{1}$-computable function $f$ the limit fails to exist. Thus, the Lebesgue differentiation theorem, in this effective setting, characterises Schnorr randomness. This converse was obtained independently by Freer et al. [19], who also extended the characterisation of Schnorr randomness to $L_{p}$-computable functions, for any fixed computable real $p \geqslant 1$.

In the meantime, Brattka, Miller and Nies proved the above-mentioned effective form of Lebesgue's theorem [31] that each nondecreasing function is a.e. differentiable: each nondecreasing computable function is differentiable at every computably random real ([6], the work for which was carried out from late 2009). Later on, Nies [43] gave a different, and somewhat simpler, argument for this result involving the geometric notion of porosity. With some extra complications the latter argument carries over to the setting of polynomial time computability, which was the main thrust of [43].

Jordan's decomposition theorem says that for every function $f$ of bounded variation there are nondecreasing functions $g_{0}, g_{1}$ such that $f=$ $g_{0}-g_{1}$. This is almost trivial in the setting of analysis (take $g_{0}(x)$ to be the variation of $f$ restricted to $[0, x]$, and let $\left.g_{1}=f-g_{0}\right)$. Thus every bounded variation function $f$ is a.e. differentiable. For computable $f$, it turns out that ML-randomness of $z$ may be required to ensure that $f^{\prime}(z)$ exists; the reason is that the two functions obtained by the Jordan decomposition cannot always be chosen to be computable. Demuth [10] had obtained results in the constructive setting which, when re-interpreted classically, show that $z$ is ML-random iff every computable function of bounded variation is differentiable at $z$. Brattka et al. [6] gave alternative proofs of both implications. For the harder implication, from left to right, they used their main result on computable nondecreasing functions, together with the fact that the possible Jordan decompositions of a computable bounded variation function form a $\Pi_{1}^{0}$ class, which therefore has a member in which $z$ is random. See the recent survey [29] for more on Demuth's work as an early example of an interplay between randomness and computability. 


\section{$5 \quad$ Lebesgue density and $K$-triviality}

Can analytic notions aid in the study of lowness via randomness? The answer is "yes, but only indirectly". Analytic notions help because they bear on our view of randomness. In this section we will review how the notion of Lebesgue density helped solving the ML-covering problem, originally asked by Stephan (2004). This was one of five "big" questions in [34]. Every c.e. set below a Turing incomplete random is a base for ML-randomness, and hence $K$-trivial [23]. The covering problem asks whether the converse holds: is every c.e. $K$-trivial $A$ below an incomplete random set? Since every $K$-trivial is Turing below a c.e. $K$-trivial [39], we can as well omit the hypothesis that $A$ be c.e.

\section{Computable analysis $\leadsto$ randomness}

Some effective versions of almost everywhere theorems lack a predefined randomness notion. In the context of Theorem 3, the statement that almost every point is a weak Lebesgue point will be called the weak Lebesgue differentiation theorem. We have already discussed the fact that the weak Lebesgue differentiation theorem for $L_{1}$-computable functions characterises Schnorr randomness. A function $g$ is lower semicomputable if $\{x: g(x)>q\}$ is $\Sigma_{1}^{0}$ uniformly in a rational $q$, and upper semicomputable if $\{x: g(x)<q\}$ is $\Sigma_{1}^{0}$ uniformly in a rational $q$. Which degree of randomness does a point $z$ need in order to ensure that $z$ is a (weak) Lebesgue point for all lower (or equivalently, all upper) semicomputable functions?

Even ML-randomness is insufficient for this. Let $z=\Omega$ denote Chaitin's halting probability, and consider the $\Pi_{1}^{0}$-set $\mathcal{C}=[\Omega, 1]$. The real $z$ is ML-random, and in particular normal: every block of bits of length $k$ (such as 110011) occurs with limiting frequency $2^{-k}$ in its binary expansion. Suppose $z \in Q$ where $Q=\left(i 2^{-n},(i+1) 2^{-n}\right)$ for some $i<n$. If the binary expansion of $z$ has a long block of $0 \mathrm{~s}$ from position $n$ on, then $\lambda(Q \cap \mathcal{C}) /|Q|$ is large; if $z$ has a long block of 1 s from $n$ on then it is small. This implies that $\lambda(Q) /|Q|$ oscillates between values close to 0 and close to 1 as $Q$ ranges over smaller and smaller basic dyadic intervals containing $z$. So it cannot be the case that $\underline{\varrho}(\mathcal{C} \mid z)=1$; in fact the density of $\mathcal{C}$ at $z$ does not exist. This means that $z$ is not a weak Lebesgue point for the upper semicomputable function $1_{\mathcal{C}}$.

We say that a ML-random real $z$ is density random if $\rho(\mathcal{C} \mid z)=1$ for each $\Pi_{1}^{0}$ set $\mathcal{C}$ containing $z$. Several equivalent characterisations of density randomness are given in [35, Thm. 5.8]; for instance, a real $z$ is density 
random iff $z$ is a weak Lebesgue point of each lower semicomputable function on $[0,1]$ with finite integral, iff $z$ is a full Lebesgue point of each such function.

\section{Randomness $\leadsto$ lowness}

The approach of the Oberwolfach group (2012) was mostly within the classical interplay of randomness and computability. Inspired by the notion of balanced randomness introduced in [18], they defined a new notion, now called Oberwolfach (OW) randomness [4]. A test notion equivalent to Oberwolfach tests, and easier to use, is as follows. A descending uniformly $\Sigma_{1}^{0}$ sequence of sets $\left\langle G_{m}\right\rangle_{m \in \omega}$, together with a left-c.e. real $\beta$ with a computable approximation $\beta=\sup _{s} \beta_{s}$, form a left-c.e. test if $\lambda G_{m}=O\left(\beta-\beta_{m}\right)$ for each $m$. Just like in the original definition of Oberwolfach tests, the test components cohere. If there is an increase $\beta_{s+1}-\beta_{s}=\gamma>0$, then all components $G_{m}$ for $m<s$ are allowed to add up to $\gamma$ in measure, as long as the sequence remains descending. We think of first $G_{0}$ adding some portion of measure of at most $\gamma$, then $G_{1}$ adding some portion of that, then $G_{2}$ a portion of that second portion, and so on up to $G_{s}$.

The Oberwolfach group [4, Thm. 1.1] showed that if $Z$ is ML-random, but not OW-random, then $Z$ computes each $K$-trivial. They also proved that OW-randomness implies density randomness.

\section{Analysis $\leadsto$ randomness $\leadsto$ lowness}

Often the notions of density are studied in the context of Cantor space $2^{\mathbb{N}}$, which is easier to work with than the unit interval. In this context one defines the density at a a bit sequence $Z$ using basic dyadic intervals that are given by longer and longer initial segments of $Z$. In the context of randomness this turns out to be a minor change. If $z$ is a ML-random real and $Z$ its binary expansion, then each $\Pi_{1}^{0}$ set $\mathcal{C} \subseteq[0,1]$ has positive density at $z$ iff each $\Pi_{1}^{0}$ set $\mathcal{C} \subseteq 2^{\mathbb{N}}$ has positive density at $Z$ iff $Z$ is Turing incomplete, by a result in Bienvenu et al. [5]. Dyadic and full density 1 also coincide for ML-random reals by a result of Khan and Miller [28, Thm. 3.12].

Day and Miller [9] used a forcing partial order specially adapted to the setting of intermediate density to prove that there is a ML-random $Z$ such that $\rho(\mathcal{C} \mid Z)>0$ for each $\Pi_{1}^{0}$ class $\mathcal{C} \subseteq 2^{\mathbb{N}}$, and at the same time there is a $\Pi_{1}^{0}$ class $\mathcal{D} \ni Z$ such that $\rho(\mathcal{D} \mid Z)<1$. Hence $Z$ is incomplete ML-random and not Oberwolfach random. By the aforementioned result 
of the Oberwolfach group [4, Thm. 1.1] this means that the single oracle $Z$ computes each $K$-trivial, thereby giving a strong affirmative answer to the covering problem.

Day and Miller also refined their argument in order to make $Z$ a $\Delta_{2}^{0}$ set. No direct construction is known to build a $\Delta_{2}^{0}$ incomplete ML-random that is not Oberwolfach random. In fact, it is open whether Oberwolfach and density randomness coincide (see Question 17 below).

\section{Cost functions and subclasses of the $K$-trivials}

In this and the following section, we survey ways to gauge the complexity of Turing degrees directly with methods inspired by analysis. The first method only applies to $K$-trivials: use the analytical tool of a cost function to study proper subideals of the ideal of $K$-trivial Turing degrees. This yields a dense hierarchy of ideals parameterised by rationals in $(0,1)$.

The second method assigns real number parameters $\Gamma(\mathbf{a}), \Delta(\mathbf{a}) \in$ $[0,1]$ to Turing degrees a in order to measure their complexity. These assignments can be interpreted in the context of Hausdorff distance in pseudometric spaces. In a sense, this second attempt turns out to be too coarse because in both variants, only the values 0 and $1 / 2$ are possible for non-computable Turing degrees (after a modification of the definition which we also present, the classes of sets with value 0 have subclasses that are potentially proper). However, this also shows that these few classes of complexity obtained must be natural and important. Although they richly interact with previously studied classes, they haven't as yet been fully characterised by other means.

Both approaches are connected to randomness through the investigations of the concepts, rather than directly through the definitions. We will explain these connections as we go along.

\section{Cost functions}

Somewhat extending [40, Section 5.3], we say that a cost function is a computable function

$$
\mathbf{c}: \mathbb{N} \times \mathbb{N} \rightarrow\{x \in \mathbb{R}: x \geqslant 0\} .
$$

For background on cost functions see [38]. We say that $\mathbf{c}$ is monotonic if $\mathbf{c}(x+1, s) \leqslant \mathbf{c}(x, s) \leqslant \mathbf{c}(x, s+1)$ for each $x$ and $s$; we also assume that $\mathbf{c}(x, s)=0$ for all $x \geqslant s$. We view $\mathbf{c}(x, s)$ as the cost of changing at stage $s$ a guess $A_{s-1}(x)$ at the value $A(x)$, for some $\Delta_{2}^{0}$ set $A$. Monotonicity means 
that the cost of a change increases with time, and that changing the guess at a smaller number is more costly.

If $\mathbf{c}$ is a cost function, we let $\mathbf{c}(x)=\sup _{s} \mathbf{c}(x, s)$. To be useful, a monotonic cost function $\mathbf{c}$ needs to satisfy the limit condition: $\underline{\mathbf{c}}(x)$ is finite for all $x$ and $\lim _{x} \mathbf{c}(x)=0$.

Definition 4 ([40]). Let $\left\langle A_{s}\right\rangle$ be a computable approximation of a $\Delta_{2}^{0}$ set $A$, and let $\mathbf{c}$ be a cost function. The total $\mathbf{c}-\operatorname{cost}$ of the approximation is

$$
\mathbf{c}\left(\left\langle A_{s}\right\rangle_{s \in \omega}\right)=\sum_{s \in \omega}\left\{\mathbf{c}(x, s): x \text { is least such that } A_{s-1}(x) \neq A_{s}(x)\right\} .
$$

We say that a $\Delta_{2}^{0}$ set $A$ obeys $\mathbf{c}$ if the total $\mathbf{c}$-cost of some computable approximation of $A$ is finite. We write $A=\mathbf{c}$.

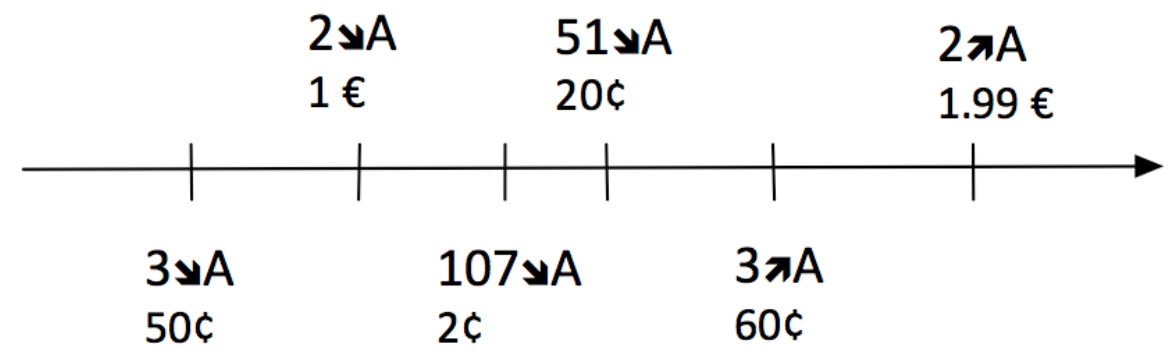

Fig. 1. Timeline illustrating the cost (in Euros) generated by an approximation of a $\Delta_{2}^{0}$ set $A$ for a particular cost function.

This definition, first given in [40], was conceived as an abstraction of the construction of a c.e. noncomputable $K$-trivial set in Downey et al. [13]. Perhaps the intuition stems from analysis. For instance, the length of a curve, i.e. a $\mathcal{C}^{1}$ function $f:[0,1] \rightarrow \mathbb{R}^{n}$, is given by $\int_{0}^{1}\left\|f^{\prime}(t)\right\| d t$. The "cost" of the change $f^{\prime}(t)$ at stage $t$ is the velocity $\left\|f^{\prime}(t)\right\|$, and to have a finite total cost means that the curve is rectifiable.

The paper [38] also treats non-monotonic cost functions, where we define $\underline{\mathbf{c}}(x)=\liminf _{s} \mathbf{c}(x, s)$ and otherwise retain the definition of the limit condition $\lim _{x} \underline{\mathbf{c}}(x)=0$. Intuitively, enumeration of $x$ into $A$ can only take place at a stage when the cost drops. This is reminiscent of $\varnothing^{\prime \prime}$ constructions, for instance building a Turing minimal pair of c.e. sets. It would be interesting to define a pair of cost functions $\mathbf{c}, \mathbf{d}$ with the limit condition such that $A \models \mathbf{c}$ and $B \models \mathbf{d}$ for c.e. sets $A, B$ imply that $A, B$ form a minimal pair. 


\section{Applications of cost functions}

Let $\beta$ be a left-c.e. real given as $\beta=\sup _{s} \beta_{s}$ for a computable sequence $\left\langle\beta_{s}\right\rangle_{s \in \omega}$ of rationals. We let $\mathbf{c}_{\beta}(x, s)=\beta_{s}-\beta_{x}$. Note that $\mathbf{c}_{\beta}$ is a monotonic cost function with the limit condition. Modifying a result from [39], in [38] it is shown that a $\Delta_{2}^{0}$ set $A$ is $K$-trivial iff $A \models \mathbf{c}_{\Omega}$. Thus $\mathbf{c}_{\Omega}$ is a cost function describing $K$-triviality. This raises the question whether obedience to cost functions stronger than $\mathbf{c}_{\Omega}$ can describe interesting subideals of the ideal of $K$-trivial Turing degrees (being a stronger cost function means being harder to obey, i.e. more expensive).

By the "halves" of a set $Z$ we mean the sets $Z_{0}=Z \cap\{2 n: n \in \mathbb{N}\}$ and $Z_{1}=Z \cap\{2 n+1: n \in \mathbb{N}\}$. If $Z$ is ML-random and $A \leqslant_{\mathrm{T}} Z_{0}, Z_{1}$ then $A$ is a base for ML-randomness, and hence $K$-trivial. So we obtain a subclass $\mathcal{B}_{1 / 2}$ of the $K$-trivial sets, namely the sets below both halves of a ML-random. Bienvenu et al. [4] had already proved that this subclass is proper. Let $\mathbf{c}_{\Omega, 1 / 2}(x, s)=\sqrt{\Omega_{s}-\Omega_{x}}$. In recent work, Greenberg, Miller and Nies obtained the following characterisation of $\mathcal{B}_{1 / 2}$.

Theorem 5 ([21], Thm. 1.1. and its proof) The following are equivalent for a set $A$.

(a) A is Turing below both halves of some $M L$-random

(b) A is Turing below both halves of $\Omega$

(c) $A$ is a $\Delta_{2}^{0}$ set that obeys $\mathbf{c}_{\Omega, 1 / 2}(x, s)$.

They generalise the result towards a characterisation of classes $\mathcal{B}_{k / n}$, where $0<k<n$. The class $\mathcal{B}_{k / n}$ consists of the $\Delta_{2}^{0}$ sets $A$ that are Turing below the effective join of any set of $k$ among the $n$-columns of some MLrandom set $Z$; as before, $Z$ can be taken to be $\Omega$ without changing the class. The characterising cost function is $\mathbf{c}_{\Omega, q}(x, s)=\left(\Omega_{s}-\Omega_{x}\right)^{q}$, where $q=k / n$. In particular, the class does not depend on the representation of $q$ as a fraction of integers. By this cost function characterisation and the hierarchy theorem [38, Thm. 3.4], $p<q$ implies that $\mathcal{B}_{p}$ is a proper subclass of $\mathcal{B}_{q}$.

Following Hirschfeldt et al. [22] we say that a set $A$ is robustly computable from a set $Z$ if $A \leqslant_{\mathrm{T}} Y$ for each set $Y$ such that the symmetric difference of $Y$ and $Z$ has upper density 0 . In [21] it is shown that the union of all the $\mathcal{B}_{q}, q<1$ rational, coincides with the sets that are robustly computable from some ML-random set $Z$. 


\section{Calibrating randomness notions via cost functions}

Bienvenu et al. [4] used cost functions to calibrate certain randomness notions. Let $\mathbf{c}$ be a monotonic cost function with the limit condition. A descending sequence $\left\langle V_{n}\right\rangle$ of uniformly c.e. open sets is a c-bounded test if $\lambda\left(V_{n}\right)=O(\underline{\mathbf{c}}(n))$ for all $n$. We think of each $V_{n}$ as an approximation for $Y \in \bigcap_{k} V_{k}$. Being in $\bigcap_{n} V_{n}$ can be viewed as a new sense of obeying $\mathbf{c}$ that works for ML-random sets. Unlike the first notion of obedience, here only the limit function $\mathbf{c}(x)$ is taken into account in the definition.

Solovay completeness is a certain universal property of $\Omega$ among the left-c.e. reals; see e.g. [12]). Using this notion, one can show that the leftc.e. bounded tests defined above are essentially the $\mathbf{c}_{\langle\Omega\rangle}$-bounded tests.

We now survey some related, as yet unpublished work of Greenberg, Miller, Nies and Turetsky dating from early 2015. Hirschfeldt and Miller in unpublished 2006 work had proven that for any $\Sigma_{3}$ null class $\mathcal{C}$ of MLrandom sets, there is a c.e. incomputable set Turing below all the members of $\mathcal{C}$. Their argument can be recast in the language of cost functions in order to show the following (here and below $\mathbf{c}$ is some monotonic cost function with the limit condition).

Proposition 6 Suppose that $A \models \mathbf{c}$ and $Y$ is in the $\Sigma_{3}^{0}$ null class of $M L$-randoms captured by a c-bounded test. Then $A \leqslant_{\mathrm{T}} Y$.

We consider sets $A$ such that the converse implication holds as well.

Definition 7. Let $A$ be a $\Delta_{2}^{0}$ set. We say that $A$ is smart for $\mathbf{c}$ if $A \models \mathbf{c}$, and $A \leqslant_{\mathrm{T}} Y$ for each $M L$-random set $Y$ that is captured by some cbounded test.

Informally, $A$ is as complex as possible for obeying $\mathbf{c}$, in the sense that the only random sets $Y$ Turing above $A$ are the ones that are above $A$ because $A$ obeys the cost function showing that $A \leqslant_{\mathrm{T}} Y$ via Proposition 6 .

For instance, $A$ is smart for $\mathbf{c}_{\Omega}$ iff no ML-random set $Y \geqslant_{T} A$ is Oberwolfach random. Bienvenu et al. [4] proved that some $K$-trivial set $A$ is smart for $\mathbf{c}_{\Omega}$. This means that $A$ is the hardest to "cover" by a MLrandom: any ML-random computing $A$ will compute all the $K$-trivials by virtue of not being Oberwolfach random.

In the new work of Greenberg et al., this result is generalised to arbitrary monotonic cost functions with the limit condition that imply $\mathbf{c}_{\Omega}$.

Theorem 8 (Greenberg et al., 2015). Let $\mathbf{c}$ be a monotonic cost function with the limit condition and suppose that only $K$-trivial sets can obey $\mathbf{c}$. Then some c.e. set $A$ is smart for $\mathbf{c}$. 
The proof of the more general result, available in [17, Part 2], is simpler than the original one. Since $A$ cannot be computable, the proof also yields a solution to Post's problem. This solution certainly has no injury, because there are no requirements.

\section{The $\Gamma$ and the $\Delta$ parameter of a Turing degree}

We proceed to our second method of gauging the complexity of Turing degrees with methods inspired by analysis. We will be able to give the intuitive notion of being "close to computable" a metric interpretation.

For $Z \subseteq \mathbb{N}$ the lower density is defined to be

$$
\underline{\eta}(Z)=\liminf _{n} \frac{|Z \cap[0, n)|}{n} .
$$

(In the literature the symbol $\rho$ is used. However, the same symbol denotes the Lebesgue density in the sense of Definition 1 , so we prefer $\eta$ here.) Hirschfeldt et al. [24] defined the $\gamma$ parameter of a set $Y$ :

$$
\gamma(Y)=\sup \{\underline{\eta}(Y \leftrightarrow S): S \text { is computable }\} .
$$

The $\Gamma$ operator was introduced by Andrews, Cai, Diamondstone, Jockusch and Lempp [1]:

$$
\Gamma(A)=\inf \left\{\gamma(Y): Y \leqslant_{T} A\right\} .
$$

It is easy to see that this only depends on the Turing degree of $A$ : one can code $A$ back into $Y$ on a sparse computable set of positions (e.g. the powers of 2), without affecting $\gamma(Y)$.

We now provide dual concepts. Let

$$
\begin{gathered}
\delta(Y)=\inf \{\underline{\eta}(Y \leftrightarrow S): S \text { computable }\}, \\
\Delta(A)=\sup \left\{\delta(Y): Y \leqslant_{T} A\right\} .
\end{gathered}
$$

Intuitively, $\Gamma(A)$ measures how well computable sets can approximate the sets that $A$ computes in the worst case (we take the infimum over all $Y \leqslant_{T} A$ ). In contrast, $\Delta(A)$ measures how well the sets that $A$ computes can approximate the computable sets in the best case (we take the supremum over all $\left.Y \leqslant_{T} A\right)$. Note that $A \leqslant_{\mathrm{T}} B$ implies $\Gamma(A) \geqslant \Gamma(B)$ and $\Delta(A) \leqslant \Delta(B)$.

It was shown in [1] that $\Gamma(A)>1 / 2 \leftrightarrow \Gamma(A)=1 \leftrightarrow A$ is computable. Clearly the maximum value of $\Delta(A)$ is $1 / 2$. It is attained, for example, when $A$ computes a Schnorr random set $Y$, because in that case $\eta(Y \leftrightarrow$ $S)=1 / 2$ for each computable $S$. Merkle, Nies and Stephan have shown that $\Delta(A)=0$ for every 2-generic $A$. 


\section{Viewing $1-\Gamma(A)$ as a Hausdorff pseudodistance}

For $Z \subseteq \mathbb{N}$ the upper density is defined by

$$
\bar{\eta}(Z)=\limsup _{n} \frac{|Z \cap[0, n)|}{n} .
$$

For $X, Y \in 2^{\mathbb{N}}$ let $d(X, Y)=\bar{\eta}(X \triangle Y)$ be the upper density of the symmetric difference of $X$ and $Y$; this is clearly a pseudodistance on Cantor space $2^{\mathbb{N}}$ (that is, two objects may have distance 0 without being equal). For subsets $\mathcal{U}, \mathcal{W}$ of a pseudometric space $(M, d)$ recall the Hausdorff pseudodistance

$$
d_{H}(\mathcal{U}, \mathcal{W})=\max \left(\sup _{u \in \mathcal{U}} d(u, \mathcal{W}), \sup _{w \in \mathcal{W}} d(w, \mathcal{U})\right)
$$

where $d(x, \mathcal{R})=\inf _{r \in \mathcal{R}} d(x, r)$ for any $x \in M, \mathcal{R} \subseteq M$. Clearly, if $\mathcal{U} \supseteq$ $\mathcal{W}$ then the second supremum is 0 , so that we only need the first. The

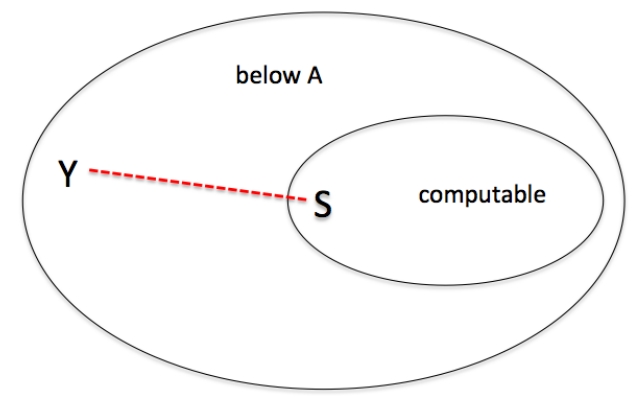

Fig. 2. Hausdorff pseudodistance $\sup _{Y \in \mathcal{A}} \inf _{S \in \mathcal{R}} d(Y, S)$.

following fact, which is now clear from the definitions, states that $1-\Gamma(A)$ gauges how close $A$ is to being computable, in the sense that it is the Hausdorff distance between the cone below $A$ and the computable sets.

Proposition 9. Given an oracle set $A$ let $\mathcal{A}=\left\{Y: Y \leqslant_{\mathrm{T}} A\right\}$. Let $\mathcal{R} \subseteq$ $\mathcal{A}$ denote the collection of computable sets. We have

$$
1-\Gamma(A)=d_{H}(\mathcal{A}, \mathcal{R})
$$

To interpret $1-\Delta(A)$ metrically, we note that $1-\delta(Y)=\sup _{S \in \mathcal{R}} d(Y, S)$. So we can view $1-\Delta(A)$ as a one-sided "dual" of the Hausdorff pseudodistance: 


$$
d_{H}^{*}(\mathcal{A}, \mathcal{R})=\inf _{Y \in \mathcal{A}} \sup _{S \in \mathcal{R}} d(Y, S) .
$$

For instance, for the unit disc $D \subseteq \mathbb{R}^{2}$ we have $d_{H}^{*}(D, D)=1$.

\section{Analogs of cardinal characteristics}

The operators $\Gamma$ and $\Delta$ are closely related to the analog in computability theory of cardinal characteristics (see [2] for the background in set theory). Both the cardinal characteristics and their analogs were introduced by Brendle and Nies in the 2015 Logic Blog [16], building on the general framework of an analogy between set theory and computability theory set up by Rupprecht ([46], also see [7]). We only discuss the versions of the concepts in the setting of computability theory.

Definition 10 (Brendle and Nies). For $p \in[0,1]$ let $\mathcal{D}\left(\sim_{p}\right)$ be the class of oracles $A$ that compute a set $X$ such that $\gamma(X) \leqslant p$, i.e., for each computable set $S$, we have $\underline{\eta}(X \leftrightarrow S) \leqslant p$.

We note that by the definitions $\Gamma(A)<p \Rightarrow A \in \mathcal{D}\left(\sim_{p}\right) \Rightarrow \Gamma(A) \leqslant p$.

Definition 11 (Brendle and Nies). Dually, for $p \in[0,1 / 2)$ let $\mathcal{B}\left(\sim_{p}\right)$ be the class of oracles $A$ that compute a set $Y$ such that for each computable set $S$, we have $\underline{\eta}(S \leftrightarrow Y)>p$.

For each $p$ we have $\Delta(A)>p \Rightarrow A \in \mathcal{B}\left(\sim_{p}\right) \Rightarrow \Delta(A) \geqslant p$.

\section{Collapse of the $\mathcal{D}\left(\sim_{p}\right)$ hierarchy for $p \neq 0$ after Monin}

Definition 12. For a computable function $h$, we let $\mathcal{D}\left(\neq_{h}^{*}\right)$, or sometimes $\mathcal{D}\left(\neq^{*}, h\right)$, denote the class of oracles $A$ that compute a function $x$ such that $\exists^{\infty} n x(n)=y(n)$ for each computable function $y<h$.

This highness notion of an oracle set $A$ was introduced by Monin and Nies in [37], where it was called " $h$-infinitely often equal". The notion also corresponds to a cardinal characteristic, namely $\mathfrak{d}\left(\neq_{h}^{*}\right)$ which is a bounded version of the well-known characteristic $\mathfrak{d}\left(\neq^{*}\right)$. The cardinal $\mathfrak{d}\left(\neq_{h}^{*}\right)$ is the least size of a set $G$ of $h$-bounded functions so that for each function $x$ there is a function $y$ in $G$ such that $\forall^{\infty} n[x(n) \neq y(n)]$. We note that $\mathcal{D}\left(\neq^{*}\right)$, i.e. the class obtained in Definition 12 when we omit the computable bound, coincides with having hyperimmune degree. See [7] for background, and in particular for motivation why the defining condition for $\mathfrak{d}\left(\neq_{h}^{*}\right)$ looks like the negation of the condition for $\mathcal{D}\left(\neq_{h}^{*}\right)$.

The proof of the following fact provides a glimpse of the methods used to prove that the $\mathcal{D}\left(\sim_{p}\right)$ hierarchy collapses. 
Proposition $13 \mathcal{D}\left(\neq^{*}, 2^{n !}\right) \subseteq \mathcal{D}\left(\sim_{0}\right)$.

Proof. Suppose that $A \in \mathcal{D}\left(\neq^{*}, 2^{n !}\right)$ via a function $x \leqslant_{\mathrm{T}} A$. Since $x(n)<$ $2^{n !}$ we can view $x(n)$ as a binary string of length $n$ !. Let $L(x) \in 2^{\mathbb{N}}$ be the concatenation of the strings $x(0), x(1), \ldots$, and let $X \leqslant \mathrm{~T} A$ be the complement of $L(x)$. Given a computable set $S$, there is a computable function $y$ with $y(n)<2^{n !}$ such that $L(y)=S$. Let $H(n)=\sum_{i<n} i$. Since $x(n)=y(n)$ for infinitely many $n$, there are infinitely many intervals $[H(n), H(n+1))$ on which $X$ and $S$ disagree completely. Since $\lim _{n} n ! / H(n)=0$ this implies $\underline{\eta}(X \leftrightarrow S)=0$. Hence $A \in \mathcal{D}\left(\sim_{0}\right)$.

We slightly paraphrase the main result of Monin's recent work [17]. It not only collapses the $\mathcal{D}\left(\sim_{p}\right)$ hierarchy, but also describes the resulting highness property combinatorially.

Theorem 14 (Monin) $\mathcal{D}(p)=\mathcal{D}\left(\neq^{*}, 2^{\left(2^{n}\right)}\right)$ for each $p \in(0,1 / 2)$. In particular, $\Gamma(A)<1 / 2 \Rightarrow \Gamma(A)=0$ so only the values 0 and $1 / 2$ can occur when $\Gamma$ is evaluated on incomputable sets.

The proof uses the list decoding capacity theorem from the theory of error-correcting codes, which says that given a sufficiently large constant $L$, a fairly large set of code words of a length $n$ can be achieved if one allows that each word of length $n$ can be close (in the Hamming distance) to up to $L$ of them. More precisely, independently of $n$, for each positive $\beta<1$ there is $L \in \omega$ so that $2^{\lfloor\beta n\rfloor}$ codewords can be achieved. (In the usual setting of error correction, one would have $L=1$, namely, each word is close to only one code word.)

\section{Collapse of the $\mathcal{B}\left(\sim_{p}\right)$ hierarchy for $p \neq 0$ via a dual of Monin}

Definition 15. For a computable function $h$, we let $\mathcal{B}\left(\neq_{h}^{*}\right)$ denote the class of oracles $A$ that compute a function $y<h$ such that $\forall^{\infty} n x(n) \neq$ $y(n)$ for each computable function $x$.

$\mathcal{B}\left(\neq^{*}\right)$, i.e. the class obtained when we omit the computable bound, coincides with "high or diagonally noncomputable" (again see, e.g., [7]). As a dual to Proposition 13 we have $\mathcal{B}\left(\sim_{0}\right) \subseteq \mathcal{B}\left(\neq^{*}, 2^{n !}\right)$.

Theorem 16 (Nies) $\mathcal{B}\left(\sim_{p}\right)=\mathcal{B}\left(\neq^{*}, 2^{\left(2^{n}\right)}\right)$ for each $p \in(0,1 / 2)$. In particular, $\Delta(A)>0 \Rightarrow \Delta(A)=1 / 2$ so there are only two possible $\Delta$ values.

For a proof see again [17]. 


\section{Open questions}

The development we have sketched in Sections 4 and 5 has led to two randomness notions. The first, density randomness, was born out of the study of randomness via computable analysis. The second, OW-randomness, was born out of the study of lowness via randomness. We know that OW-randomness implies density randomness.

Question 17. Do OW-randomness and density randomness coincide?

One direction of attack to answer this negatively could be to look at other properties of points that are implied by OW-randomness, and show that density randomness does not suffice. By [35, Thm. 6.1] OW-randomness of $z$ implies the existence of the limit in the sense of the Birkhoff ergodic theorem (Section 4) for computable operators $T$ on a computable probability space $\left(2^{\mathbb{N}}, \mu\right)$, and lower semicomputable functions $g: X \rightarrow \mathbb{R}$. For another example, by [20] OW-randomness of $z$ also implies an effective version of the Borwein-Ditor theorem: if $\left\langle r_{i}\right\rangle_{i \in \omega}$ is a computable null sequence of reals and $z \in \mathcal{C}$ for a $\Pi_{1}^{0}$ set $\mathcal{C} \subseteq \mathbb{R}$, then $z+r_{i} \in \mathcal{C}$ for infinitely many $i$.

Lowness for density randomness coincides with $K$-triviality by [35, Thm. 2.6]. Lowness for OW randomness is merely known to imply $K$ triviality for the reasons discussed in Section 3; further, an incomputable c.e. set that is low for OW-randomness has been constructed in unpublished worked with Turetsky.

Question 18. Characterise lowness for OW-randomness. Is it the same as $K$-triviality?

Section 7 leaves open several questions.

Question 19. Is $\mathcal{D}\left(\sim_{0}\right)$ a proper subclass of $\mathcal{D}\left(\neq^{*}, 2^{\left(2^{n}\right)}\right)=\mathcal{D}(1 / 4)$ ? Is $\mathcal{D}\left(\neq^{*}, 2^{n !}\right)$ a proper subclass of $\mathcal{D}\left(\neq^{*}, 2^{\left(2^{n}\right)}\right)$ ?

By Proposition 13 an affirmative answer to the first part implies an affirmative answer to the second. The dual open questions are:

Question 20. Is $\mathcal{B}\left(\neq^{*}, 2^{\left(2^{n}\right)}\right)=\mathcal{B}\left(\sim_{0.25}\right)$ a proper subclass of $\mathcal{B}\left(\sim_{0}\right)$ ? Is it a proper subclass of $\mathcal{B}\left(\neq^{*}, 2^{(n !)}\right)$ ?

\section{Acknowledgement}

Most of the research surveyed in this article was supported by the Marsden Fund of New Zealand. 


\section{References}

1. U. Andrews, M. Cai, D. Diamondstone, C. Jockusch, and S. Lempp. Asymptotic density, computable traceability, and 1-randomness. Preprint, 2013.

2. T. Bartoszyński and Haim Judah. Set Theory. On the structure of the real line. A K Peters, Wellesley, MA, 1995. 546 pages.

3. L. Bienvenu, A. Day, N. Greenberg, A. Kučera, J. Miller, A. Nies, and D. Turetsky. Computing $K$-trivial sets by incomplete random sets. Bull. Symb. Logic, 20:80-90, 2014.

4. L. Bienvenu, N. Greenberg, A. Kučera, A. Nies, and D. Turetsky. Coherent randomness tests and computing the K-trivial sets. To appear in J. European Math. Society, 2016

5. L. Bienvenu, R. Hölzl, J. Miller, and A. Nies. Denjoy, Demuth, and Density. J. Math. Log., 1450004, 2014. 35 pages.

6. V. Brattka, J. Miller, and A. Nies. Randomness and differentiability. Transactions of the AMS, 368:581-605, 2016. arXiv version at http://arxiv.org/abs/1104. 4465 .

7. J. Brendle, A. Brooke-Taylor, Keng Meng Ng, and A. Nies. An analogy between cardinal characteristics and highness properties of oracles. In Proceedings of the 13th Asian Logic Conference: Guangzhou, China, pages 1-28. World Scientific, 2013. http://arxiv.org/abs/1404.2839.

8. G. Chaitin. Information-theoretical characterizations of recursive infinite strings. Theoretical Computer Science, 2:45-48, 1976.

9. A. R. Day and J. S. Miller. Density, forcing and the covering problem. Mathematical Research Letters, 22(3):719-727, 2015.

10. O. Demuth. The differentiability of constructive functions of weakly bounded variation on pseudo numbers. Comment. Math. Univ. Carolin., 16(3):583-599, 1975. (Russian).

11. R. Downey and D. Hirschfeldt. Algorithmic randomness and complexity. SpringerVerlag, Berlin, 2010. 855 pages.

12. R. Downey, D. Hirschfeldt, and A. Nies. Randomness, computability, and density. SIAM J. Comput., 31(4):1169-1183, 2002.

13. R. Downey, D. Hirschfeldt, A. Nies, and F. Stephan. Trivial reals. In Proceedings of the 7th and 8th Asian Logic Conferences, pages 103-131, Singapore, 2003. Singapore University Press.

14. R. Downey, A. Nies, R. Weber, and L. Yu. Lowness and $\Pi_{2}^{0}$ nullsets. J. Symbolic Logic, 71(3):1044-1052, 2006.

15. R. G. Downey and Carl G. Jockusch, Jr. T-degrees, jump classes, and strong reducibilities. Trans. Amer. Math. Soc., 30:103-137, 1987.

16. A. Nies (editor). Logic Blog 2015. Available at http://arxiv.org/abs/1602. 04432, 2015.

17. A. Nies (editor). Logic Blog 2016. Available at cs.auckland.ac.nz/ nies, 2016.

18. Santiago Figueira, Joseph S. Miller, and André Nies. Indifferent sets. J. Logic Comput., 19(2):425-443, 2009.

19. C. Freer, B. Kjos-Hanssen, A. Nies, and F. Stephan. Algorithmic aspects of Lipschitz functions. Computability, 3(1):45-61, 2014.

20. A. Galicki and A. Nies. Effective Borwein-Ditor theorem. Proceedings of CiE 2016, to appear.

21. N. Greenberg, J. Miller, and A. Nies. A dense hierarchy of subideals of the $k$-trivial degrees. 
22. D. Hirschfeldt, Jockusch C., R. Kuyper, and P. Schupp. Coarse reducibility and algorithmic randomness. arXiv preprint arXiv:1505.01707, 2015.

23. D. Hirschfeldt, A. Nies, and F. Stephan. Using random sets as oracles. J. Lond. Math. Soc. (2), 75(3):610-622, 2007.

24. Denis R Hirschfeldt, Carl G Jockusch Jr, Timothy McNicholl, and Paul E Schupp. Asymptotic density and the coarse computability bound. Preprint, 2013.

25. C. G. Jockusch, Jr. The degrees of bi-immune sets. Z. Math. Logik Grundlagen Math., 15:135-140, 1969.

26. Carl G. Jockusch, Jr. Semirecursive sets and positive reducibility. Trans. Amer. Math. Soc., 131:420-436, 1968.

27. Carl G. Jockusch, Jr. and Robert I. Soare. $\Pi_{1}^{0}$ classes and degrees of theories. Trans. Amer. Math. Soc., 173:33-56, 1972.

28. M. Khan. Lebesgue density and $\Pi_{1}^{0}$-classes. J. Symb. Logic, To appear.

29. A. Kučera, A. Nies, and C. Porter. Demuth's path to randomness. Bull. Symb. Logic, 21(3):270-305, 2015.

30. H. Lebesgue. Leçons sur l'Intégration et la recherche des fonctions primitives. Paris: Gauthier-Villars, 1904.

31. H. Lebesgue. Sur les intégrales singulières. Ann. Fac. Sci. Toulouse Sci. Math. Sci. Phys. (3), 1:25-117, 1909.

32. H. Lebesgue. Sur l'intégration des fonctions discontinues. Ann. Sci. École Norm. Sup. (3), 27:361-450, 1910.

33. D. A. Martin and W. Miller. The degrees of hyperimmune sets. Z. Math. Logik Grundlag. Math., 14:159-166, 1968.

34. J. S. Miller and A. Nies. Randomness and computability: Open questions. Bull. Symbolic Logic, 12(3):390-410, 2006.

35. K. Miyabe, A. Nies, and J. Zhang. Using almost-everywhere theorems from analysis to study randomness. Bull. Symb. Logic, to appear, 2016.

36. J. Mohrherr. A refinement of $\operatorname{low}_{n}$ and high $n$ for the r.e. degrees. Z. Math. Logik Grundlag. Math., 32(1):5-12, 1986.

37. B. Monin and A. Nies. A unifying approach to the Gamma question. In Proceedings of Logic in Computer Science (LICS). IEEE press, 2015.

38. A. Nies. Calculus of cost functions. To appear in Barry Cooper and Mariya Soskova (eds.), The Incomputable: Journeys beyond the Turing barrier, Springer-Verlag.

39. A. Nies. Lowness properties and randomness. Adv. in Math., 197:274-305, 2005.

40. A. Nies. Computability and randomness, volume 51 of Oxford Logic Guides. Oxford University Press, Oxford, 2009. 444 pages. Paperback version 2011.

41. A. Nies. Interactions of computability and randomness. In Proceedings of the International Congress of Mathematicians, pages 30-57. World Scientific, 2010.

42. A. Nies. Studying randomness through computation. In Randomness through computation, pages 207-223. World Scientific, 2011.

43. A. Nies. Differentiability of polynomial time computable functions. In Ernst W. Mayr and Natacha Portier, editors, 31st International Symposium on Theoretical Aspects of Computer Science (STACS 2014), volume 25 of Leibniz International Proceedings in Informatics (LIPIcs), pages 602-613, Dagstuhl, Germany, 2014. Schloss Dagstuhl-Leibniz-Zentrum fuer Informatik.

44. N. Pathak. A computational aspect of the Lebesgue differentiation theorem. $J$. Log. Anal., 1:Paper 9, 15, 2009.

45. N. Pathak, C. Rojas, and S. G. Simpson. Schnorr randomness and the Lebesgue differentiation theorem. Proc. Amer. Math. Soc., 142(1):335-349, 2014. 
46. Nicholas Rupprecht. Effective correspondents to cardinal characteristics in Cichon's diagram. PhD thesis, University of Michigan, 2010.

47. Gerald E. Sacks. A minimal degree below 0'. Bull. Amer. Math. Soc., 67:416-419, 1961.

48. Gerald E. Sacks. The recursively enumerable degrees are dense. Ann. of Math. (2), 80:300-312, 1964.

49. C.P. Schnorr. Zufälligkeit und Wahrscheinlichkeit. Eine algorithmische Begründung der Wahrscheinlichkeitstheorie. Springer-Verlag, Berlin, 1971. Lecture Notes in Mathematics, Vol. 218.

50. R. Solovay. Handwritten manuscript related to Chaitin's work. IBM Thomas J. Watson Research Center, Yorktown Heights, NY, 215 pages, 1975.

51. C. Spector. On the degrees of recursive unsolvability. Ann. of Math. (2), 64:581$592,1956$.

52. S. Terwijn and D. Zambella. Algorithmic randomness and lowness. J. Symbolic Logic, 66:1199-1205, 2001.

53. V. V'yugin. Ergodic theorems for individual random sequences. Theor. Comput. Sci., 207(2):343-361, 1998. 